\title{
The role of guidance and counseling in promoting student discipline in secondary schools in Kenya: A case study of Kisumu district
}

\author{
Article by Farzana Begum \\ School Of MSN, Texila American University, Kenya \\ Email:-mehrbanfarz@gmail.com
}

\section{Source}

Jack O. Ajowi and Enose M. W. Simatwa, 'The role of guidance and counseling in promoting student discipline in secondary schools in Kenya: A case study of Kisumu district', Education Research and Reviews, Vol. 5 (5), pp. 263-272, May, 2010, http://www.academicjournals.org/article/article1379608450_Ajowi\%20and\%20Simatwa.pdf

\section{Introduction}

This is the review which critiques on the article 'The role of guidance and counseling in promoting student discipline in secondary schools in Kenya: A case study of Kisumu district' which was published in the Journal "Education Research and Reviews", "Vol. 5 (5), pp. 263272, May, 2010". During the review it will include the summary of this article. It 'll also depict the structure analysis by giving the feedback on it that how well it has been written with respect to the understanding and readability of the reader. There will be the analysis on the way of elaborating that how well it is providing the information that has been used in it. The review will also contain the critique on the article along with evaluating the authority, its currency, accuracy, objectivity and coverage as well.

\section{Review of Literature}

\section{Article Summary}

The article is providing the analysis on guidance and counseling services, activities, attitudes and relationships so that as much as possible, each pupil's basic psychological needs which are satisfied through the medium of education. It mentions five basic questions on which the whole research depends and concludes.

\section{Article Structure}

The article contains the proper structure which is very intuitive for the reader. It starts with an introduction which gives a proper idea about the article to the reader even before reading the full article and the reader can judge whether the article is of interest or not. It includes very distinct sections and provides proper references. It contains a tree like structure with proper headings and helping reader to directly move to the section of interest. References section contains the clear references in text.

\section{Article Critique}

\section{Authority}

The article was published in the journal "Education Research and Reviews", "Vol. 5 (5), pp. 263-272, May, 2010" is a peer reviewed open access journal. It is published twice monthly and also covers all areas of the subjects such as comparative education, early childhood education, adult education, special education, teacher's training, vocation education, educational technology, educational administration and management, curriculum development, education policies and management etc.

The authors have to met the requirements of the editorial policy and publication ethics then the the article can be published in this journal. This makes it very credible source for the articles and hence adding the worth to the published articles.

\section{Accuracy}


South American Journal of Academic Research

Special Edition May 2016

The accuracy of this article can be seen in the sources of information mentioned in the article and making it very authentic. It provides very comprehensive information to making its accuracy more clear. All the information provided contains the proper references too, as increasing the accuracy.

\section{Currency}

The journal issue containing the article was published in 2010, while the article was accepted for publishing in March 16, 2010. It shows that the research is current and article cites up-to-date references.

\section{Relevance}

The journal in which this article was published contains the academic context. It is concerned with promoting the guidance and counseling hence is highly related to nursing.

\section{Objectivity}

The article contains the information which depicts the research and has been objectively directed. The authors have good research work and their expertise adding more insight for the readers. The findings show that guidance and counseling has not been effectively used to promote student discipline. This was evidenced in the disciplinary cases that were found in the schools. According to this research article, the understanding about the importance of the guidance and counseling is of worth. It could be more influencing if it could have some statistical background and references too. Otherwise, it shows the strength of this research.

\section{Stability}

The article was published in a journal which contains very stable data so making itself a stable source.

\section{Analysis of graph/Image/Table}

(Not Applicable)

\section{Conclusion}

This review critiques and also provides the summary of the article very intuitively. According to this article certain administrative tools such as effective supervision, effective leadership and effective communication are vital tools in ensuring the role of guidance and counseling in promoting student discipline. 